\title{
Índice de Valor Paisagístico para árvores em ambiente urbano
}

\author{
MAURICIO ESTELLITA ${ }^{(2)}$ e MARIAESMERALDASOARES PAYÃO DEMATTÊ(1)
}

\begin{abstract}
RESUMO
No ambiente urbano, freqüentemente, ocorrem conflitos entre arborização, construções e redes aéreas de serviços públicos, o que tem levado à erradicação de árvores, por ser, quase sempre, a solução mais simples e de menor custo monetário. Com esse procedimento, podem ser destruídos indivíduos arbóreos de valor para a comunidade e cuja integridade deveria ser preservada, por meio de sua classificação como imunes ao corte, de acordo com seu valor paisagístico. O objetivo deste trabalho foi, a partir de um índice já existente, o Iir (Índice de Importância Relativa), formular um novo índice, o IVP (Índice de Valor Paisagístico), destinado a atribuir valores para a importância de indivíduos arbóreos urbanos como integrantes do patrimônio paisagístico da cidade, testando-se o modelo. Os dados obtidos no teste resultaram de medições e avaliações de 20 árvores do centro da cidade de Jaboticabal( SP). Foi usada a fórmula: IVP = [(4 Ve) + (2 Vc) + (3 VL) + (5 Vbm) + Vec + (10 Vhc) $]$ / Abr, em que: IVP= Índice de Valor Paisagístico; Ve = valor da espécie (raridade, origem, velocidade de crescimento, disponibilidade e adaptação); Vc = valor de condição (estado sanitário geral); VL = valor de localização (adequação do indivíduo ao local onde se encontra); Vbm = valor biométrico (relacionado com as dimensões do indivíduo); Vec = valor ecológico (benefícios ecológicos); Vhc = valor histórico e cultural; Abr = abundância relativa da espécie (número de indivíduos da espécie em relação ao número total de indivíduos arbóreos na cidade). Os resultados indicaram coerência com as observações empíricas iniciais, e a conclusão do presente trabalho é de que o IVP, na forma aqui descrita, presta-se a uma avaliação eficiente do valor paisagístico de indivíduos arbóreos para sua eventual inclusão como árvores imunes ao corte.
\end{abstract}

Palavras-chaves: arborização urbana, patrimônio paisagístico, avaliação, Índice de Valor Paisagístico (IVP), teste de modelo.

\begin{abstract}
Index for estimation of trees landscaping value in urban environment.

In urban environments, conflicts between trees and sidewalks, buildings and aerial public service lines often occur, leading to tree eradications, because this is generally the simplest and the cheapest solution. With this procedure, important trees for the local communities can be destroyed, instead of being protected by law because of their value in the landscape. Here, the objective was to formulate and to test an index for evaluating the landscaping value of trees in urban environments, the so called Index of Landscaping Value (ILV), based on the Index of Relative Importance already used. The ILV is proposed for estimating with numeric values the importance of individual urban trees in the context of the landscaping heritage of the town. Twenty trees located in the center of Jaboticabal, São Paulo, Brazil, were evaluated in the test, using the formula: ILV $=[(4 \mathrm{SV}+(2 \mathrm{CV})+(3 \mathrm{LV})+(5 \mathrm{BV})+\mathrm{EV}+(10 \mathrm{HCV})] /$ Abr, where: ILV= Index of Landscape Value; SV = species value (rarity, origin, growth speed, availability and adaptability); $\mathrm{CV}=$ condition value (general sanitary condition); $\mathrm{LV}=$ localization value (adequacy to the place where the tree grows); $\mathrm{BV}=$ biometric value (related to tree dimensions); $\mathrm{EV}=$ ecological value (ecological benefits); HCV = historic and cultural values; Abr = relative abundance (number of individuals of the species in relation to the total number of individuals of all tree species in the town). Comparing the results of the ILV calculation with the initial empiric observations, it was concluded that this index could be used efficiently as an evaluating method of the landscaping value of urban trees aiming at their eventual inclusion as trees protected by law.
\end{abstract}

Key words: urban trees, landscape patrimony, evaluation, Index of Landscaping Value (ILV), model test.

\section{INTRODUÇÃO}

Freqüentemente, as administrações municipais defrontam-se com situações em que é preciso decidir entre cortar uma árvore ou tomar medidas para que os problemas causados por sua localização sejam solucionados de outro modo, em geral mais dispendioso. Nessas ocasiões, às vezes, não é levado em conta o valor da árvore que a diferencia de outras e, que com seu corte, perdem-se precioso elemento estético e ecológico que, entre outros benefícios, contribui para o interesse turístico da cidade e para a conservação de sua memória.

Muitos municípios brasileiros têm, em seu perímetro urbano, árvores que, por alguns atributos especiais, são valiosas como patrimônio. É importante que esses indivíduos vegetais, nativos ou exóticos, sejam reconhecidos pelo público e recebam tratamento especial: leis que os protejam de corte, ou imunidade ao corte, na expressão jurídica; cuidados para deter ataque de pragas ou doenças; adequação do espaço que ocupam, para que possam permanecer em bom estado e exercer plenamente seu efeito.

\footnotetext{
(1) Trabalho extraído da Dissertação de Mestrado do primeiro autor.

(2) Centro Universitário Barão de Mauá, Curso de Arquitetura e Urbanismo. Rua Chile, 845,

1o andar, 14020-610, Ribeirão Preto (SP).

(3) Departamento de Produção Vegetal, Faculdade de Ciências Agrárias e Veterinárias, Unesp, Câmpus de Jaboticabal. Via de Acesso Prof.

Paulo Donato Castellane, S/N., 14884-900, Jaboticabal (SP) - Brasil. Bolsista do CNPq - e-mail: inga.ambiental@netsite.com.br
} 
Outra questão que tem sido discutida é o valor monetário de indivíduos que fazem parte da arborização urbana, com a finalidade de estabelecer multas a quem lhes infringir danos ou morte.

Inicialmente, o principal critério para avaliar o valor de uma árvore urbana, do ponto de vista econômicomonetário, segundo GREY \& DENEKE (1978) e MILANO \& DALCIN (2000), era o da circunferência ou área transversal atingida pelo tronco; portanto, a avaliação era associada ao valor da madeira.

DALCIN (1992) elaborou um modelo matemático para avaliação de indivíduos arbóreos em coleções botânicas vivas, levando em consideração variáveis inerentes à espécie, ao local e ao indivíduo, e sua raridade na comunidade. Com base nesse modelo, SILVA FILHO (2002) propôs índices para valoração de árvores urbanas e os aplicou à cidade de Jaboticabal, (SP) entre esses índices, está o de Importância Relativa (SILVA FILHO et al., 2002).

É hipótese deste trabalho que o Índice de Importância Relativa, proposto por DALCIN (1992) e aplicado por SILVA FILHO et al. (2002), embora tenha uso principal na avaliação do valor monetário de determinada árvore, pode ser adaptado para avaliar sua importância paisagística, pois contempla diversos critérios considerados para escolha dos indivíduos arbóreos a serem protegidos. Por isso, o objetivo deste trabalho foi o de propor uma modificação desse índice e de testar sua aplicabilidade para avaliar o valor de indivíduos arbóreos notáveis dentro do patrimônio paisagístico do centro de Jaboticabal (SP), assim como aferir valores de referência.

\section{MATERIALEMÉTODOS}

O Índice de Valor Paisagístico (IVP), proposto no presente trabalho para valoração patrimonial de árvores urbanas, baseou-se no Índice de Importância Relativa (Iir) proposto por DALCIN (1992) e aplicado por SILVA FILHO et al. (2002):

$\operatorname{Iir}=($ Ve x Vc x VL x Vbm) / Freq, em que:

$\mathrm{Ve}=$ valor da espécie (relacionado com disponibilidade ou raridade, utilidade, desenvolvimento e adaptabilidade do indivíduo);

Vc = valor de condição (estado geral do indivíduo);

$\mathrm{VL}=$ valor de localização (relacionado com adequação ao local onde o indivíduo se encontra);

Vbm = valor biométrico (relacionado com as dimensões do indivíduo);

Freq $=$ porcentagem de indivíduos da espécie em relação ao número total de indivíduos na arborização local.

As modificações realizadas no Iir neste trabalho, para determinar o IVP, consistiram, especialmente, na inclusão de componentes relacionados com os critérios considerados na escolha de indivíduos imunes ao corte: origem da espécie, como componente da soma representada por valor da espécie (Ve), valor ecológico (Vec) e valor histórico e cultural (Vhc). Foram também atribuídos pesos diferentes aos diversos componentes, com a intenção de valorizar os atributos mais relacionados com o objetivo do trabalho, o de avaliar a contribuição paisagística de cada indivíduo. A inclusão desses componentes na fórmula implicou alteração do valor relativo dos demais e, para obter resultados numéricos menores e de mais fácil percepção, optou-se por adicioná-los, em vez de multiplicá-los.

Procurou-se uma fórmula de cálculo em que uma hipotética árvore que alcançasse a avaliação máxima atingisse um valor de IVP próximo a 100, e outra que alcançasse a avaliação mínima recebesse um valor o mais próximo possível de 1 . Por esse sistema, as árvores avaliadas variam em uma faixa de IVP $\cong 1$ a IVP $=100$, facilitando o entendimento da grandeza de IVP atingida por cada indivíduo.

Indivíduos mortos não são avaliados pelo IVP.

A fórmula adaptada e desenvolvida especialmente para este trabalho é a seguinte:

$\mathrm{IVP}=[(4 \mathrm{Ve})+(2 \mathrm{Vc})+(3 \mathrm{VL})+(5 \mathrm{Vbm})+\mathrm{Vec}+(10$

Vhc)]/ Abr, em que:

$\mathrm{Ve}=$ valor da espécie (com origem, disponibilidade ou raridade, presença ou não de princípios tóxicos ou alergênicos, desenvolvimento e adaptabilidade do indivíduo);

$\mathrm{Vc}=$ valor de condição (estado geral do indivíduo);

$\mathrm{VL}=$ valor de localização (adequação do indivíduo ao local onde se encontra);

Vbm = valor biométrico (relacionado com as dimensões do indivíduo);

Vec $=$ valor ecológico (relacionado com benefícios ecológicos adicionais àqueles já proporcionados pela presença da arborização no meio urbano, como redução da temperatura ambiente, aumento da umidade relativa do ar, redução do número de partículas em suspensão na atmosfera e abrigo à fauna, e outros, que, por si só, a vegetação proporciona). Como benefícios adicionais, foram considerados: proteção de solo sujeito a erosão, sombreamento proporcionado por copa de grande diâmetro, alimento proporcionado à avifauna e a outros pequenos animais, produção de sementes viáveis, integração em conjunto arbóreo, suporte às plantas epífitas;

Vhc = valor histórico e cultural;

Abr = abundância relativa (número de indivíduos da espécie em relação ao número total de indivíduos de todas as espécies, na arborização da cidade); este componente foi representado por notas.

As notas atribuídas a cada componente da fórmula foram adaptadas de SILVA FILHO et al. (2002), ou, nos casos de inclusão de novos componentes, foram criadas.

As de valor da espécie (Ve) são constituídas conforme a expressão que segue.

$\mathrm{Ve}=$ (origem + disponibilidade + toxicidade + desenvolvimento + adaptabilidade) / 5, sendo:

- origem: nota 1 = espécie exótica de ocorrência freqüente em arborização urbana regional, originária de comunidades vegetais diferentes daquelas da região em estudo; 2 = espécie exótica de ocorrência pouco freqüente em arborização urbana regional, originária de comunidades vegetais diferentes daquelas da região em estudo; 3 = espécie nativa do Brasil ou ocorrente em formações florestais semelhantes as do local de estudo, mesmo que estejam situadas em países limítrofes; 4 = espécie nativa do Brasil e ocorrente na flora regional;

- disponibilidade: 1 = mudas disponíveis no local ou na quantidade desejada; 2 = encontradas com relativa 
facilidade; 3 = mudas encontradas com dificuldade; reprodução difícil; 4 = mudas não encontradas no mercado e/ou técnica de reprodução desconhecida ou não realizada; espécie ameaçada de extinção;

- toxicidade: 1 = partes tóxicas e/ou frutos tóxicos ao alcance dos transeuntes; 2 = princípios alergênicos; 3 = toxicidade leve; 4 = sem toxicidade relatada;

- desenvolvimento: 1 = rápido; 2 = medianamente rápido; 3 = lento; 4 = muito lento;

- adaptabilidade: 1= espécie espontânea na região; 2 = espécie de fácil adaptação; 3 = espécie exigente; 4 = espécie de difícil adaptação, muito exigente.

As notas atribuídas ao valor de condição $(\mathrm{Vc})$ obedecem aos seguintes valores: 1 = indivíduo danificado com possibilidade de recuperação após tratamento; 2 = indivíduo com danos leves; 3 = indivíduo sem danos aparentes; 4 = indivíduo sadio com ótima aparência.

As notas para valor de localização (VL) são: $0=$ inadequação (impedimento físico que induz a procedimentos de manutenção como podas drásticas e/ ou que somente pode ser solucionado com reformas onerosas); 1 = com necessidade de adequação do espaço que pode ser solucionada com pequenas reformas e podas periódicas leves; 2 = com necessidade de adequação do espaço que pode ser solucionada com pequenas reformas; 3 = com necessidade de adequação do espaço que pode ser solucionada com podas periódicas leves; $4=0$ indivíduo é adequado ao espaço ocupado.

O valor biométrico (Vmb) é calculado com a seguinte fórmula:

Vbm $=[(0,3$ DAP $)+(0,3 \mathrm{H})+(0,2 \mathrm{DC})] / 3$, em que:

$\mathrm{DAP}=$ diâmetro do tronco à altura do peito $(1,30 \mathrm{~m}$ de altura a partir da superfície do solo); $\mathrm{H}=$ altura do ponto mais alto da árvore; DC = diâmetro maior da copa; as medidas são expressas em metros.

Com referência ao valor ecológico (Vec), as notas são as seguintes: 1 = sem função ecológica especial, além dos benefícios que toda árvore proporciona; 2 = com um a dois benefícios ecológicos adicionais proporcionados ao ambiente urbano; 3 = com três a quatro benefícios ecológicos adicionais proporcionados ao ambiente urbano; 4 = com cinco ou mais benefícios ecológicos adicionais proporcionados ao ambiente urbano.

O valor histórico e cultural (Vhc) é avaliado pelas seguintes notas: 1 = sem referência; 2 = valor histórico e/ou cultural do indivíduo ou do conjunto em que se integra citado em literatura especializada ou documentos históricos (nessas informações, são também consideradas aquelas relacionadas com a importância da árvore dos pontos de vista medicinal, ornamental, alimentício ou de madeira e matérias-primas); 3 = o indivíduo ou o conjunto em que se integra tem significado especial para a comunidade local, manifestado em depoimentos verbais, observações e/ou documentos escritos; 4 = o indivíduo ou o conjunto em que se integra tem valor histórico e/ou cultural citado em literatura especializada ou documentos históricos e tem significado especial para a comunidade local. O componente Vhc foi valorizado na fórmula em relação aos demais, por se considerar que uma árvore com valor histórico-cultural ou com significado especial para a comunidade local, somente por este atributo, é de grande valor.
As notas usadas para representar a abundância relativa (Abr) são: 1 = menor que 1\%; 2 = entre 1,0 e 9,9\%; $3=$ entre 10 e $15 \% ; 4$ = maior que $15 \%$.

\subsection{Teste do Índice de Valor Paisagístico (IVP) proposto}

O IVP proposto foi testado para avaliar árvores previamente escolhidas no centro de Jaboticabal (SP), cuja localização é apresentada na tabela 1.

A cidade de Jaboticabal, no Estado de São Paulo, situa-se a $21^{\circ} 15^{\prime}$ de latitude sul e $48^{\circ} 18^{\prime}$ ' de longitude oeste. O município abrange 708,6 km², com 33 km² de área urbana e população de 67.389 habitantes. Informações históricas, geográficas, socioeconômicas e culturais sobre Jaboticabal são encontradas em FREITAS (1978), CAPALBO (1978 e 1997), POLI (1986), ANDRADE (2000), JABOTICABAL (2002) e PEGOLO \& DEMATTÊ (2002).

A área de estudo, definida como Bairro Centro da cidade de Jaboticabal, de acordo com o considerado pela Prefeitura Municipal, abrange um quadrilátero formado pelas ruas Juca Quito e Floriano Peixoto em confluência com as avenidas Major Novais e General Glicério.

Nessa área, foram escolhidas árvores existentes no espaço público que se enquadravam em critérios que as caracterizam como exemplares merecedores de proteção legal visando à sua preservação. A lista foi iniciada com as árvores anteriormente selecionadas por VALERI et al. (2000).

A escolha dos demais indivíduos arbóreos foi feita com base nos critérios considerados pela legislação brasileira para declarar uma árvore imune ao corte (DIREITO AMBIENTAL, 2004; GOVERNO DO ESTADO DO ESPÍRITO SANTO, 2004; PREFEITURA MUNICIPALDE CURITIBA, 2004; PREFEITURA MUNICIPAL DE JOINVILLE, 2004; PREFEITURA MUNICIPAL DE SALVADOR, 2004): localização (dos pontos de vista de proteção do solo, sombreamento que proporciona, integração em conjunto de valor paisagístico, integração em conjunto de interesse histórico); contribuição à qualidade ambiental; origem (são mais valorizadas as espécies da flora local); raridade (do ponto de vista botânico, tratando-se de espécie ameaçada de extinção ou pouco conhecida localmente; do ponto de vista cultural, por suas características curiosas ou incomuns); porte (indivíduo que atingiu grande tamanho quando comparado com outros de sua espécie); beleza; condição de portasementes; antiguidade e interesse histórico; interesse científico e cultural; interesse paisagístico; significado especial para a comunidade local.

A seguir, foram entrevistadas pessoas da cidade, tanto ligadas à memória do patrimônio paisagístico, como moradores antigos do centro de Jaboticabal: Abdo Messias Neme, Clovis Capalbo, Brasilina Morelli, Dorival Martins de Andrade, Edith Marinelli Oliveira, Levy Martins do Lago e Osvaldo Ligeiro. As entrevistas foram conduzidas informalmente, abordando-se fatos de interesse histórico e cultural sobre as árvores escolhidas.

As informações sobre cada exemplar escolhido abrangeram também a localização exata do indivíduo, sua identificação botânica, altura, DAP, diâmetro basal, estado sanitário e adequação ao espaço circundante. 
Foram também consideradas as informações de literatura especializada (CORRÊA, 1926 a 1978; HOEHNE, 1944; SANCHOTENE, 1985; ANDERSEN \& ANDERSEN, 1988; LORENZI, 1992 e 1998; LORENZI et al., 1996; CARAUTA\& DIAZ, 2002; LORENZI et al., 2003), incluindo características botânicas, origem, utilização e possível risco de extinção. Para complementar a pesquisa sobre o valor histórico local desses indivíduos, anteriormente levantado nas entrevistas, foram utilizadas informações de WHITAKER (1978), AQUINO (1983), PINTO (1989), LUPINACCI \& PINTO (1992); MARCHIORI et al. (1992); ANDRADE (2000) e DEMATTÊ (2002).

Serviram de base, para avaliar a abundância relativa (Abr), as espécies da arborização urbana de Jaboticabal listadas nos trabalhos de CABRIL (2001), que fez menção àquelas existentes nas principais praças, e de SILVA FILHO (2002), que realizou cadastramento da arborização viária.

Para aferição de valores de referência, foram introduzidos dois indivíduos hipotéticos, um com as notas máximas possíveis e com grandes dimensões (grande Vbm), designado por A, e outro com as notas mínimas possíveis e Vbm mínimo, chamado de B. No cálculo do Vbm do indivíduo $\mathbf{B}$, consideraram-se os seguintes valores mínimos para uma árvore: DAP de 0,10 m (conforme muitos autores, entre os quais OLIVEIRA E SANT’ANNA, 2003); altura máxima de quatro metros e diâmetro máximo da copa de dois metros (MARTO et al., 2006).

\section{RESULTADOS E DISCUSSÃO}

As árvores avaliadas pelo índice proposto neste trabalho e os respectivos valores atribuídos estão listados na tabela 2. A fórmula para cálculo do IVP dos indivíduos escolhidos não indicou grandes problemas de aplicabilidade, e os valores encontrados não foram discordantes da avaliação empírica inicial. Os critérios usados para o cálculo do IVP produziram um formato de resultados que permite fácil compreensão do valor do IVP, com a introdução dos dois indivíduos hipotéticos; o indivíduo $\mathbf{A}$, que recebeu as notas máximas possíveis, atingiu IVP $=100$, e ao $\mathbf{B}$, com as notas mínimas possíveis, foi atribuído IVP = 4,92 (o valor mais próximo possível de 1).

As árvores escolhidas inicialmente, consideradas importantes como patrimônio paisagístico, atingiram valores de IVP entre 79,60 e 44,70. Assim, numa primeira aproximação, pode-se estabelecer que um valor de IVP acima de 40,00 indica uma árvore que deveria ser declarada imune ao corte.

As modificações para adequar o Índice de Importância Relativa (Iir), proposto por DALCIN (1992) e aplicado por SILVA FILHO et al. (2002), à avaliação do valor patrimonial paisagístico de indivíduos arbóreos foram feitas com base nos critérios considerados pela legislação brasileira para declarar uma árvore imune ao corte, por causa da semelhança de propósitos. Assim, foram incluídos o valor ecológico (Vec) e o valor histórico e cultural (Vhc). No fator valor da espécie (Ve) foi incluído um componente para valorizar as espécies nativas, especialmente as representativas da flora regional.

No fator Ve da fórmula usada por SILVA FILHO et al. (2002), um dos componentes relaciona-se com partes da árvore desejáveis ou indesejáveis. Esse componente foi retirado no IPV aqui proposto, porque partes indesejáveis estão relacionadas com localização, que já é considerada em outro fator (VL). As raízes de um flamboyant, por exemplo, são indesejáveis em arborização viária e junto a construções e pisos, mas em espaços gramados de praças e parques não apresentam inconvenientes e produzem belo efeito.

No que se refere ao valor de condição (Vc), considerou-se que um indivíduo arbóreo, quando danificado, pode receber tratamento que proporcione sua recuperação (BARCELOS, 2000; WEBB et al., 2002). Árvores mortas, na fórmula usada por SILVA FILHO et al. (2002), recebem nota zero, resultando em valor zero para o Índice de Importância Relativa (Iir). No Índice de Valor Paisagístico aqui proposto, o critério é semelhante, já que indivíduos mortos não são incluídos na avaliação.

Com referência ao valor de localização (VL), uma mesma espécie pode ser adequada para determinado local e inadequada para outro. Uma espécie com sistema radicular abundante e superficial pode ter alto VL se for localizada em um parque, e baixo VL se localizada em uma calçada, fazendo parte da arborização viária. Entretanto, em qualquer caso, o critério aqui adotado foi o mesmo de SILVA FILHO et al. (2002): o valor de localização nunca deve reduzir o índice de importância, e sim acrescentar, quando ocorrerem condições favoráveis, evitando que, por exemplo, a presença de fiação ou outro equipamento urbano seja um elemento detrator de um espécime arbóreo, reduzindo seu valor. Na aplicação do Iir (SILVA FILHO et al., 2002), em que se multiplicam os fatores, a nota zero (localização inadequada, com restrições severas) foi substituída por 1 , de modo que não se acrescenta valor de localização ao resultado final. No IVP aqui proposto, em que os componentes são somados, a nota para localização inadequada, com restrições severas, é zero, resultando também em não-atribuição de valor adicional ao resultado final.

Uma das dificuldades encontradas no presente trabalho refere-se à expressão do Vbm (valor biométrico) apenas em função das dimensões do indivíduo, sem levar em consideração os padrões da espécie a que pertence. Dessa forma, a idade da árvore e o ambiente em que ela se desenvolveu são os principais fatores que influenciam esse componente. Pode haver outras causas do maior ou menor desenvolvimento de uma árvore, como a variabilidade genética dentro da espécie. Entretanto, um indivíduo pertencente a uma espécie de pequeno porte, mesmo que tenha dimensões superiores aos padrões médios dessa espécie, terá sempre um valor biométrico inferior ao de um indivíduo de espécie de grande porte, ainda que este último apresente, em relação ao padrão médio de sua espécie, dimensões reduzidas. O ideal seria que o Vmb fosse estimado por notas resultantes da comparação das medidas do indivíduo avaliado com as de um indivíduo-padrão de sua espécie, informação esta não-disponível de forma consistente na literatura especializada.

Por outro lado, árvores de grandes dimensões despertam o interesse do observador de modo especial, e isso representa valor paisagístico.

A distorção causada pelo problema de aferição do Vbm no resultado final do cálculo do Índice de Valor 
Paisagístico (IVP) é diminuída pela inclusão dos outros componentes da fórmula. Comparando a espécie de menor porte, uma jabuticabeira (Myrciaria trunciflora), com a de maior porte, uma figueira (Ficus sp.), com valores respectivos de Vbm de 0,92 e 4,06, verifica-se que a jabuticabeira teve maior IVP (Tabela 2). Esse resultado deve-se aos maiores valores de Ve, VL e Vhc da jabuticabeira, quando comparados com os da figueira.

Outra limitação do uso, tanto do Iir, como do IVP, está na necessidade de se conhecer, na arborização urbana local, a porcentagem de indivíduos de uma determinada espécie em relação ao número total de indivíduos de todas as espécies (componente chamado de Freq no Iir e de Abr no IVP). No caso de Jaboticabal, não há levantamento das árvores existentes em praças e outras áreas verdes do perímetro urbano, exceto o levantamento florístico realizado no Bosque Municipal por LUPPINACI \& PINTO (1992), referente ao remanescente florestal nativo. A solução encontrada foi tomar por base o cadastramento da arborização viária de Jaboticabal feito por SILVA FILHO (2002) e avaliar se a porcentagem da espécie, calculada por esse autor, era semelhante ou diferente da observada nas principais praças da cidade e em todas as praças da área em estudo. Dentro dos limites estabelecidos para cada nota, verificou-se que o valor de abundância relativa (Abr) para determinada espécie, com base nas porcentagens calculadas por SILVA FILHO (2002), era aplicável a toda a área urbana, pois em Jaboticabal, em geral, não existem grandes diferenças entre a arborização viária e a de praças. Assim, o cálculo do IVP foi possível.

Com referência às notas estabelecidas para Abr, esta é outra modificação do IVP em comparação com o Iir usado por SILVA FILHO et al. (2002). No Iir, o valor de Freq é representado diretamente pela porcentagem calculada (porcentagem de árvores da espécie considerada em relação ao número total de espécies presentes na arborização urbana). No IVP, estabeleceram-se limites de porcentagem para atribuição das notas, por causa da dificuldade em obter valores exatos, já que não foram feitos levantamentos mais detalhados. Os limites de 10 a $15 \%$ fundamentaram-se em MILANO \& DALCIN (2000); esses autores consideraram que a porcentagem de determinada espécie na arborização de uma cidade não deve ultrapassar essa faixa.

A exigência de formação técnica do avaliador para a aplicação de índices de valoração deve ser levada em conta.

Para aplicação do IVP, é preciso treinamento. Na atribuição de notas de valor ecológico (Vec), por exemplo, deve ficar claro o que se entende por benefícios ecológicos proporcionados por qualquer árvore e benefícios ecológicos adicionais, como foi especificado em Material e Métodos, na conceituação desse componente. Além disso, serão necessárias tabelas ou fichas, elaboradas por especialistas, com informações sobre árvores ornamentais. Uma contribuição significativa tem sido dada por DALCIN (2006). Entre as informações mais importantes estão a origem da espécie e a formação vegetal em que ela ocorre.

A beleza, um valor, até certo ponto subjetivos, mencionados na legislação como atributos a ser considerados em árvores imunes ao corte, estão incluídos em componentes do IVP que podem ser avaliados objetivamente. Pode ser representada por árvores de grande porte, com grande valor biométrico (Vbm), e por indivíduos sadios, sem sinais de danos (alto Vc).

A origem da espécie (em Ve), o valor ecológico(Vec) e o valor histórico e cultural (Vhc) têm também significado importante dos pontos de vista turístico, educativo e psicológico.

Um sonho? Tenho sim. Tive um sonho mais abrangente que fantasiava uma bela praça em torno da velha Figueira... Agora sonho menor. Um pequeno espaço reservado ao seu entorno, um espaço verde que não precisa ter mais do que uns 10 metros quadrados, mas que seja gramado, cuidado e protegido. E que tenha também alguns dizeres que mostrem aos passantes o que significou a velha árvore.

Essa é uma história que tomou corpo nas minhas noites insones, quando as saudades são maiores e só pensamentos sobre algo simbólico, importante e querido amenizam um pouco a dor de uma ausência.

(Trechos de "A história de uma árvore", Neuza Guerreiro de CARVALHO, 2004).

\section{CONCLUSÃO}

O IVP(Índice de Valor Paisagístico), na forma aqui descrita, presta-se a uma avaliação eficiente do valor paisagístico de indivíduos arbóreos para sua eventual classificação como árvores imunes ao corte.

\section{REFERÊNCIAS}

ANDERSEN, O.; ANDERSEN, V. U. As frutas silvestres brasileiras. Rio de Janeiro: Globo, 1988. 203p.

ANDRADE, D. M. Jaboticabal, jabuticabeira, jabuticaba: achegas para o conhecimento. Jaboticabal: Funep, 2000. 122p.

AQUINO, C. A. de. Projeto de reflorestamento de áreas selecionadas da Faculdade de Ciências Agrárias e Veterinárias do Câmpus de Jaboticabal da Universidade Estadual Paulista. 1983. 28f. Monografia (Trabalho de Graduação em Agronomia) - Faculdade de Ciências Agrárias e Veterinárias, Universidade Estadual Paulista, Jaboticabal.

BARCELOS, P. R. A. Manejo da arborização urbana. In: CONGRESSO BRASILEIRO DE ARBORIZAÇÃO URBANA, 5., Rio de Janeiro, 2000. (Apostila de mini-curso).

CABRIL, L. C. N. Estudo sobre as principais praças de Jaboticabal e Taquaritinga (SP). 2001. 113f. Dissertação (Mestrado em Conservação e Manejo de Recursos, Área de Gestão Integrada de Recursos) - Centro de Estudos Ambientais, Universidade Estadual Paulista, Rio Claro.

CAPALBO, C. R. A história de Jaboticabal - 1828-1978. Jaboticabal: edição do autor, 1978. 532p.

CAPALBO, C. R. A história de Jaboticabal - 1979-1997e outras histórias (1979-1997). Jaboticabal: edição do autor, 1997. 505p. 
CARAUTA, J. P. P.; DIAZ, B. E. Figueiras no Brasil. Rio de Janeiro: Editora da Universidade Federal do Rio de Janeiro, 2002. 208p.

CARVALHO, N. G. de. Ahistória de uma árvore. Disponível em: <http://planeta.terra.com.br/educacao/fdg/ historia_arvore.htm>. Acesso em: 1 abr.2004.

CORRÊA, M. P. Dicionário das plantas úteis do Brasil e das exóticas cultivadas. Rio de Janeiro: Imprensa Nacional, Ministério da Agricultura, Indústria e Comércio, 1926 a 1978. 6 volumes.

DALCIN, E. C. Base de dados colaborativa sobre árvores ornamentais no Brasil. Disponível em: $<\underline{\text { http:// }}$

www.dalcin.org/arvores/

index.php?title=P\%C3\%A1gina_principal $>$. Acesso em: 9jul.2006.

DALCIN, E. C. Índice de Importância Relativa (Iir) e Valor da Espécie (Ve): proposta de uma fórmula para avaliar exemplares arbóreos na arborização urbana. In: CONGRESSO BRASILEIRO DE ARBORIZAÇÃO URBANA, 1., 1992, Vitória. Anais... Vitória: Sociedade Brasileira de Arborização Urbana, 1992. p.291-305.

DEMATTÊ, M. E. S. P. Vegetação regional em arborização urbana: estudo sobre Jaboticabal (SP). In: CONGRESSO BRASILEIRO DE ARBORIZAÇÃO URBANA, 6., 2002, Goiânia. Anais... Goiânia: Sociedade Brasileira de Arborização Urbana, 2002. 1 CD-Rom.

DIREITO AMBIENTAL. Disponível em: <http:// www.aultimaarcadenoe.com.br/lei4771.htm>. Acesso em: 1 abr.2004.

FREITAS, S. O. de. (Ed.). Jaboticabal, SP-Brasil: edição do sesquicentenário. São Paulo: Edições Populares, 1978. 100p.

GOVERNO DO ESTADO DO ESPÍRITO SANTO. Lei no 4.701, de 01 de dezembro de 1992. Disponível em: $<$ http:/ /www.seama.es.gov.br/scripts/legislacao/leis/ lei_4701.asp>. Acesso em: 1 abr.2004.

GREY, G. W.; DENEKE, F. J. Urban forestry. New York: John Wiley, 1978. 279p.

HOEHNE, F. C. Arborização urbana. São Paulo: Secretaria da Agricultura, Indústria e Comércio, 1944. 215p.

JABOTICABAL. Disponível em <http:// www.jaboticabal.com.br>. Acesso em: 7 fev. 2002.

LORENZI, H. Árvores brasileiras: manual de identificação e cultivo de plantas arbóreas nativas do Brasil. Nova Odessa: Editora Plantarum, 1992. 352p.

LORENZI, H. Árvores brasileiras: manual de identificação e cultivo de plantas arbóreas nativas do Brasil. Nova Odessa: Editora Plantarum, 1998. v.2, 352p.

LORENZI, H.; SOUZA, H. M.; MEDEIROS-COSTA, J. T. de; CERQUEIRA, L. S. C. de; BEHR, N. von. Palmeiras no Brasil: nativas e exóticas. Nova Odessa: Editora Plantarum, 1996. 303p.

LORENZI, H.; SOUZA, H. M.; TORRES, M. A. V.; BACHER,
L. B. Árvores exóticas no Brasil: madeireiras, ornamentais e aromáticas. Nova Odessa, Instituto Plantarum, 2003. 368p.

LUPINACCI, A. M.; PINTO, J. B. Relação de espécies identificadas no Bosque Municipal Francisco Buck. In: PREFEITURA MUNICIPAL DE JABOTICABAL. Levantamento arbóreo do Bosque Municipal Francisco Buck. Jaboticabal, 1992. Cap.3. Não paginado.

MARCHIORI, A. C. C.; NASSAR JUNIOR, F.; DEMATTÊ, M. E. S. P. Levantamento florístico e fitossociológico do estrato arbóreo de mata residual do Viveiro Experimental da FCAV-Unesp, Jaboticabal, SP. In: CONGRESSO DA SOCIEDADE BOTÂNICA DE SÃO PAULO, 8., 1990, Campinas. Anais... Campinas: Sociedade Botânica de São Paulo, 1992. p.121-131.

MARTO, G B. T.; BARRICHELO, L. E. G; SILVAFILHO, D. F. da; MÜLLER, P. H. Arborização urbana. Disponível em: $<$ http://www.ipef.br/silvicultura/arborizacaourbana.asp>. Acesso em: 14 jul..2006.

MILANO, M. S.; DALCIN, E. C. Arborização de vias públicas. Rio de Janeiro: Light, 2000. 226p.

OLIVEIRA, M. V. N. d'; SANT’ANNA, H. Inventário florestal e avaliação do avanço do desmatamento no projeto de colonização Pedro Peixoto. Rio Branco: Embrapa Acre, 2003. 47p. (Documentos, 83).

PEGOLO, L. C. N.; DEMATTÊ, M. E. S. P. Estudo sobre as principais praças de Jaboticabal e Taquaritinga (SP). Holos Environment, Rio Claro, v.2, n.1, p.106-123, 2002. Disponível em http://www.rc.unesp.br/ib/cea/holos. Acesso em: 8 set.2002.

PINTO, M. M. Levantamento fitossociológico de mata residual situada no Câmpus de Jaboticabal da Unesp. 1989. 114f. Dissertação (Mestrado em Agronomia, Área de Produção Vegetal) - Faculdade de Ciências Agrárias e Veterinárias, Universidade Estadual Paulista, Jaboticabal, 1989.

POLI, F. N. Listagem básica dos bens culturais relevantes situados no centro comercial - Jaboticabal, SP. 1986. 231f. Monografia (Trabalho de Graduação) - Curso de Arquitetura e Urbanismo, Instituição Moura Lacerda, Ribeirão Preto, 1986.

PREFEITURA MUNICIPAL DE CURITIBA. Árvores históricas ganham mais proteção em Curitiba. Disponível em: <http://www.curitiba.pr.gov.br/pmc/ agencia/conteudo.asp?npag=1\&ncod=1238 $>$. Acesso em: 11 abr.2004.

PREFEITURA MUNICIPAL DE JOINVILLE. Lei Complementar no 29, de 14 de junho de 1996. Disponível em: <http://www.arvore.com.br/artigos/arquivos/ Codigo_Municipal_do_Meio_ambiente.doc $>$. Acesso em: 1 abr.2004.

PREFEITURA MUNICIPALDE SALVADOR. Decreto $\mathbf{n}^{\circ}$ 5.674 de 19 de junho de 1979. Disponível em: $<\underline{\text { http:} / /}$ www.seplam.pms.ba.gov.br/legisla/legurban/D567479_topo.htm>. Acesso em 1 abr.2004.

SANCHOTENE, M. do C. C. Frutíferas nativas úteis à 
fauna na arborização urbana. PortoAlegre: Feplam, 1985. 311p.

SILVA FILHO, D. F. da. Cadastramento informatizado, sistematização e análise da arborização das vias públicas da área urbana do município de Jaboticabal, SP. 2002. 81f. Dissertação (Mestrado em Agronomia, Área de Produção Vegetal) - Faculdade de Ciências Agrárias e Veterinárias, Universidade Estadual Paulista, Jaboticabal, 2002.

SILVAFILHO, D. F. da; PIZETTA, P. U. C.; ALMEIDA, J. B. S. A. de; PIVETTA, K. F. L.; FERRAUDO, A. S. Banco de dados relacional para cadastro, avaliação e manejo da arborização em vias públicas. Revista Árvore, Viçosa, v.26, n.5, p.629-642, 2002.

VALERI, S. V.; PIVETTA, K. F. L.; DEMATTÊ, M. E. S. P.
Árvores imunes ao corte no município de Jaboticabal, SP. In: CONGRESSO BRASILEIRO DE ARBORIZAÇÃO URBANA, 5., 2000, Rio de Janeiro. Anais... Rio de Janeiro: Sociedade Brasileira de Arborização Urbana, 2000. 1 CDRom.

WEBB, P. J.; CHURATA, C. M.; DEMATTÊ, M. E. S. P. Dendrocirurgia com argila. In: CONGRESSO BRASILEIRO DEARBORIZAÇÃO URBANA, 6., 2002, Goiânia. Anais... Goiânia: Sociedade Brasileira de Arborização Urbana, 2002. 1CD-Rom.

WHITAKER, A. P. de A. Os primeiros anos de uma história sesquicentenária. In: FREITAS, S. O. de. (Ed.). Jaboticabal, SP - Brasil: edição do sesquicentenário. São Paulo: Edições Populares, 1978. p.11-23.

Tabela 1. Localização das árvores avaliadas no centro de Jaboticabal (SP)

\begin{tabular}{|c|c|c|c|c|c|}
\hline \multicolumn{3}{|c|}{ Árvore ${ }^{(1)}$} & \multirow{2}{*}{ Espécie } & \multirow{2}{*}{\begin{tabular}{l}
\multicolumn{1}{c}{ Nome regional } \\
Tipuana (conjunto da R. \\
Gen. Osório)
\end{tabular}} & \multirow{2}{*}{$\begin{array}{l}\text { Local } \\
\begin{array}{l}\text { R. General Osório, em frente ao no } 202 \text {, lado } \\
\text { norte. }\end{array}\end{array}$} \\
\hline $\mathrm{AV}$ & 1 & $\mathrm{C}$ & & & \\
\hline $\mathrm{AV}$ & 1 & $\mathrm{D}$ & Tipuana tipu & $\begin{array}{l}\text { Tipuana (conjunto da R. } \\
\text { Gen. Osório) }\end{array}$ & R. General Osório, em frente ao no 202, lado sul. \\
\hline $\mathrm{AV}$ & 3 & & $\begin{array}{l}\text { Caesalpinia } \\
\text { pelthoporoides }\end{array}$ & Sibipiruna & $\begin{array}{l}\text { R. Floriano Peixoto, esquina com a Av. Major } \\
\text { Novaes. }\end{array}$ \\
\hline PHM & 1 & & Ficus microcarpa & Ficus & $\begin{array}{l}\text { Praça Dom José M. Homem de Mello, em frente } \\
\text { ao } \mathrm{n}^{\mathrm{0}} 18 .\end{array}$ \\
\hline PJB & 4 & & Cedrela fissilis & Cedro & $\begin{array}{l}\text { Praça Dr. Joaquim Batista, em frente à Biblioteca } \\
\text { Pública. }\end{array}$ \\
\hline PJB & 5 & & Hymenaea courbaril & Jatobá & Praça Dr. Joaquim Batista, em frente ao no150. \\
\hline PJB & 3 & A & Ficus sp. & $\begin{array}{l}\text { Figueira (conjunto da } \\
\text { praça da Matriz) }\end{array}$ & $\begin{array}{l}\text { Praça Dr. Joaquim Batista, em frente à R. São } \\
\text { Sebastião. }\end{array}$ \\
\hline PJB & 3 & $\mathrm{~B}$ & Ficus sp. & $\begin{array}{l}\text { Figueira (conjunto da } \\
\text { praça da Matriz) }\end{array}$ & $\begin{array}{l}\text { Praça Dr. Joaquim Batista, em frente à Biblioteca } \\
\text { Pública. }\end{array}$ \\
\hline PJB & 6 & & Genipa americana & Jenipapo & Praça Dr. Joaquim Batista, em frente ao no 108. \\
\hline PJB & 7 & & Tamarindus indica & Tamarindo & Praça Dr. Joaquim Batista, em frente ao no 40. \\
\hline PJB & 8 & $\mathrm{~F}$ & Licania tomentosa & $\begin{array}{l}\text { Oiti (conjunto da Av. } \\
\text { Major Novaes) }\end{array}$ & Praça Dr. Joaquim Batista, em frente ao nº 51. \\
\hline PJB & 8 & G & Licania tomentosa & $\begin{array}{l}\text { Oiti (conjunto da Av. } \\
\text { Major Novaes) }\end{array}$ & Praça Dr. Joaquim Batista, em frente ao nº 69. \\
\hline PJB & $\begin{array}{l}1 \\
1\end{array}$ & & Myroxylon peruiferum & Cabriúva & Praça Dr. Joaquim Batista, em frente ao no 115. \\
\hline PJB & $\begin{array}{l}1 \\
2\end{array}$ & & Delonix regia & Flamboyant & Praça Dr. Joaquim Batista, em frente ao nº 197. \\
\hline PJB & $\begin{array}{l}1 \\
4\end{array}$ & & Sapindus saponaria & Sabão-de-soldado & Praça Dr. Joaquim Batista, em frente ao no 273. \\
\hline PJB & $\begin{array}{l}1 \\
5\end{array}$ & & Cedrela fissilis & $\begin{array}{l}\text { Cedro (cedro-dos- } \\
\text { pracinhas) }\end{array}$ & $\begin{array}{l}\text { Praça Dr. Joaquim Batista, monumento no centro } \\
\text { da praça. }\end{array}$ \\
\hline PJT & 1 & $\mathrm{D}$ & $\begin{array}{l}\text { Tabebuia } \\
\text { impetiginosa }\end{array}$ & $\begin{array}{l}\text { Ipê-roxo (conjunto da } \\
\text { R. General Osório) }\end{array}$ & Praça Jorge Tibiriçá, em frente ao no 84 . \\
\hline
\end{tabular}


Tabela 1. continuação

\begin{tabular}{|c|c|c|c|c|c|}
\hline \multicolumn{3}{|c|}{ Árvore ${ }^{(1)}$} & \multirow{2}{*}{$\frac{\text { Espécie }}{\text { Tabebuia impetiginosa }}$} & \multirow{2}{*}{$\begin{array}{l}\text { Nome regional } \\
\text { Ipê-roxo (conjunto da R. } \\
\text { General Osório) }\end{array}$} & \multirow{2}{*}{$\begin{array}{l}\text { Local } \\
\begin{array}{l}\text { R. General Osório, esquina com Av. Barão } \\
\text { do Rio Branco.' }\end{array}\end{array}$} \\
\hline PJT & 1 & $\mathrm{E}$ & & & \\
\hline PJT & 3 & & Jacaranda mimosifolia & Jacarandá-mimoso & $\begin{array}{l}\text { Praça Jorge Tibiriçá, em frente ao no } 448 \\
\text { da Av. Barão do Rio Branco. }\end{array}$ \\
\hline PNJ & 2 & & Myrciaria trunciflora & Jabuticabeira & Praça Nove de Julho, em frente ao n⿳o 32 . \\
\hline
\end{tabular}

(1) Localização: AV (Arborização viária ); PHM (Praça Dom José Marcondes Homem de Mello); PJB (Praça Dr. Joaquim Batista); PJT (Praça Jorge Tibiriçá), e PNJ (Praça Nove de Julho);

1 a 15 são números que as árvores receberam em uma mesma localização;

letras de A a G referem-se à posição da árvore dentro de um conjunto de árvores da mesma espécie.

Tabela 2. Índice de Valor Paisagístico (IVP) de árvores do centro de Jaboticabal (SP)

\begin{tabular}{|c|c|c|c|c|c|c|c|c|c|c|}
\hline Árvore & Espécie & Nome regional & $\mathrm{Ve}^{(1)}$ & Vc & VL & Vbm* & Vec & Vhc & Abr & $\operatorname{IVP}^{(2)}$ \\
\hline$A^{* *}$ & & & 4,00 & 4,00 & 4,00 & 4,00 & 4,00 & 4,00 & 1,00 & 100,00 \\
\hline PNJ 2 & $\begin{array}{l}\text { Myrciaria } \\
\text { trunciflora }\end{array}$ & Jabuticabeira & 3,00 & 4,00 & 4,00 & 0,92 & 3,00 & 4,00 & 1,00 & 79,60 \\
\hline PJB 15 & Cedrela fissilis & $\begin{array}{l}\text { Cedro (cedro-dos- } \\
\text { pracinhas) }\end{array}$ & 2,20 & 1,00 & 4,00 & 2,27 & 3,00 & 4,00 & 1,00 & 77,15 \\
\hline PJB 3 A & Ficus sp. & $\begin{array}{l}\text { Figueira } \\
\text { (conjunto da praça } \\
\text { da Matriz) }\end{array}$ & 2,40 & 4,00 & 2,00 & 4,06 & 3,00 & 3,00 & 1,00 & 76,90 \\
\hline PHM 1 & Ficus microcarpa & Ficus & 2,00 & 4,00 & 3,00 & 2,81 & 1,00 & 3,00 & 1,00 & 70,05 \\
\hline PJT $1 \mathrm{E}$ & $\begin{array}{l}\text { Tabebuia } \\
\text { impetiginosa }\end{array}$ & $\begin{array}{l}\text { Ipê-roxo } \\
\text { (conjunto da R. } \\
\text { General Osório) }\end{array}$ & 2,60 & 4,00 & 1,23 & 3,00 & 3,00 & 3,00 & 1,00 & 69,55 \\
\hline PJT 1 D & $\begin{array}{l}\text { Tabebuia } \\
\text { impetiginosa }\end{array}$ & $\begin{array}{l}\text { Ipê-roxo } \\
\text { (conjunto da R. } \\
\text { General Osório) }\end{array}$ & 2,60 & 4,00 & 1,07 & 3,00 & 3,00 & 3,00 & 1,00 & 68,75 \\
\hline PJB 3 B & Ficus sp. & $\begin{array}{l}\text { Figueira } \\
\text { (conjunto da praça } \\
\text { da Matriz) }\end{array}$ & 2,40 & 4,00 & 2,00 & 2,39 & 3,00 & 3,00 & 1,00 & 68,55 \\
\hline PJB 5 & $\begin{array}{l}\text { Hymenaea } \\
\text { courbaril }\end{array}$ & Jatobá & 3,00 & 4,00 & 4,00 & 2,36 & 4,00 & 2,00 & 1,00 & 67,80 \\
\hline PJB 6 & Genipa americana & Jenipapo & 2,60 & 4,00 & 4,00 & 2,51 & 4,00 & 2,00 & 1,00 & 66,95 \\
\hline PJB 7 & Tamarindus indica & Tamarindo & 2,40 & 4,00 & 4,00 & 2,44 & 4,00 & 2,00 & 1,00 & 65,80 \\
\hline PJB 11 & $\begin{array}{l}\text { Myroxylon } \\
\text { peruiferum }\end{array}$ & Cabriúva & 3,00 & 4,00 & 4,00 & 2,06 & 3,00 & 2,00 & 1,00 & 65,30 \\
\hline PJT 3 & $\begin{array}{l}\text { Jacaranda } \\
\text { mimosifolia }\end{array}$ & Jacarandá-mimoso & 2,40 & 2,00 & 3,00 & 2,13 & 1,00 & 3,00 & 1,00 & 64,25 \\
\hline PJB 4 & Cedrela fissilis & Cedro & 2,20 & 4,00 & 4,00 & 2,48 & 2,00 & 2,00 & 1,00 & 63,20 \\
\hline PJB 14 & $\begin{array}{l}\text { Sapindus } \\
\text { saponaria }\end{array}$ & Sabão-de-soldado & 2,80 & 4,00 & 4,00 & 1,51 & 1,00 & 2,00 & 1,00 & 59,75 \\
\hline AV 1 D & Tipuana tipu & $\begin{array}{l}\text { Tipuana } \\
\text { (conjunto da R. } \\
\text { Gen. Osório) }\end{array}$ & 2,40 & 4,00 & 0,00 & 1,57 & 2,00 & 3,00 & 1,00 & 57,45 \\
\hline AV $1 \mathrm{C}$ & Tipuana tipu & $\begin{array}{l}\text { Tipuana } \\
\text { (conjunto da R. } \\
\text { Gen. Osório) }\end{array}$ & 2,40 & 4,00 & 0,00 & 1,07 & 2,00 & 3,00 & 1,00 & 54,95 \\
\hline
\end{tabular}


Tabela 2. continuação

\begin{tabular}{|c|c|c|c|c|c|c|c|c|c|c|}
\hline Árvore & Espécie & Nome regional & $\mathrm{Ve}^{(1)}$ & Vc & VL & Vbm* & Vec & Vhc & Abr & $\operatorname{IVP}^{(2)}$ \\
\hline PJB 12 & Delonix regia & Flamboyant & 2,00 & 2,00 & 2,00 & 2,17 & 3,00 & 2,00 & 1,00 & 51,85 \\
\hline AV 3 & $\begin{array}{l}\text { Caesalpinia } \\
\text { peltophoroides }\end{array}$ & Sibipiruna & 2,40 & 4,00 & 3,00 & 1,90 & 2,00 & 2,00 & 2,00 & 48,10 \\
\hline PJB 8 F & $\begin{array}{l}\text { Licania } \\
\text { tomentosa }\end{array}$ & $\begin{array}{l}\text { Oiti } \\
\text { (conjunto da Av. } \\
\text { Major Novaes) }\end{array}$ & 2,60 & 4,00 & 3,00 & 1,46 & 3,00 & 3,00 & 4,00 & 45,20 \\
\hline PJB 8 G & $\begin{array}{l}\text { Licania } \\
\text { tomentosa }\end{array}$ & $\begin{array}{l}\text { Oiti } \\
\text { (conjunto da Av. } \\
\text { Major Novaes) }\end{array}$ & 2,60 & 4,00 & 3,00 & 1,36 & 3,00 & 3,00 & 4,00 & 44,70 \\
\hline $\mathbf{B}^{* *}$ & & & 1,00 & 1,00 & $\mathbf{0 , 0 0}$ & 0,54 & 1,00 & 1,00 & 4,00 & 4,92 \\
\hline
\end{tabular}

(1) Ve: valor de espécie (relacionado com origem, disponibilidade ou raridade, presença ou não de princípios tóxicos ou alergênicos, desenvolvimento e adaptabilidade da árvore).

Vc: valor de condição (estado geral da árvore).

VL: valor de localização (adequação da árvore ao local onde se encontra).

Vbm: valor biométrico (relacionado com as dimensões da árvore).

Vec: valor ecológico (relacionado com a função ecológica da árvore no ambiente urbano).

Vhc: valor histórico e cultural.

Abr: abundância relativa da espécie na arborização local (número de indivíduos da espécie em relação ao número total de indivíduos arbóreos na cidade).

(2) $\mathrm{IVP}=[(4 \mathrm{Ve})+(2 \mathrm{Vc})+(3 \mathrm{VL})+(5 \mathrm{Vbm})+\mathrm{Vec}+(10 \mathrm{Vhc})] / \mathrm{Abr}$

* Valores não atribuídos por notas, mas sim, por medições.

** Indivíduos hipotéticos com valores máximos (A) e mínimos (B) fictícios. 